\title{
A NONLINEAR PARABOLIC PROBLEM WITH SINGULAR TERMS AND NONREGULAR DATA
}

\author{
FRANCESCANTONIO OLIVA AND FRANCESCO PETITTA
}

ABSTRACT. We study existence of nonnegative solutions to a nonlinear parabolic boundary value problem with a general singular lower order term and a nonnegative measure as nonhomogeneous datum, of the form

$$
\begin{cases}u_{t}-\Delta_{p} u=h(u) f+\mu & \text { in } \Omega \times(0, T), \\ u=0 & \text { on } \partial \Omega \times(0, T), \\ u=u_{0} & \text { in } \Omega \times\{0\},\end{cases}
$$

where $\Omega$ is an open bounded subset of $\mathbb{R}^{N}(N \geq 2)$, $u_{0}$ is a nonnegative integrable function, $\Delta_{p}$ is the $p$-laplace operator, $\mu$ is a nonnegative bounded Radon measure on $\Omega \times(0, T)$ and $f$ is a nonnegative function of $L^{1}(\Omega \times(0, T))$. The term $h$ is a positive continuous function possibly blowing up at the origin. Furthermore, we show uniqueness of finite energy solutions in presence of a nonincreasing $h$.

\section{CONTENTS}

1. Introduction

Notations.

2. Setting and main existence result

3. Existence of a distributional solution

3.1. Approximation scheme and a priori estimates

3.2. Passing to the limit

3.3. Some comments on the regularity of the solutions

4. Uniqueness of finite energy solutions

Acknowledgements

References

\section{INTRODUCTION}

Let $\Omega$ be an open bounded subset of $\mathbb{R}^{N}$; we are mainly concerned with nonnegative solutions of problems modeled by

$$
\begin{cases}u_{t}-\Delta_{p} u=f u^{-\gamma}+\mu & \text { in } \Omega \times(0, T), \\ u=0 & \text { on } \partial \Omega \times(0, T), \\ u=u_{0} & \text { in } \Omega \times\{0\},\end{cases}
$$

where $p>2-\frac{1}{N+1}, u_{0}$ is a nonnegative integrable function, $\mu$ is a nonnegative bounded Radon measure on $\Omega \times(0, T), f$ is a nonnegative function in $L^{1}(\Omega \times(0, T))$, and $\gamma>0$.

The interest in problems as 1.1 (with $p=2$ and smooth data) started in [18 in connection with the study of thermo-conductivity ( $u^{\gamma}$ represented the resistivity of the material), and later in the study of signal transmissions and in the theory non-Newtonian pseudoplastic fluids (28 27 19]).

From the purely mathematical point of view, a complete setting of the theory, in the stationary case with smooth data, was developed over the years starting by the seminal papers [37 10, until the remarkable improvements given in in [22] $(p=2$ and $\mu=0)$.

2010 Mathematics Subject Classification. 35K10, 35K20, 35K65, 35K67, 35R06.

Key words and phrases. Singular parabolic problems; Existence and uniqueness; Measure data. 
Again in the stationary case with $p=2$ the weak theory was settled in [6] (see also [7 20, 21)) while both existence and uniqueness in presence of nonlinear operators was proven in 8 30 31 38. The case of possibly measure as data was faced in $30,35,13$.

In the parabolic setting the literature for problems as in 1.1 is, by far, more limited. If $f \equiv 0$ (the case quasilinear case with measure data) we refer to [33. (see also [16, 32 34]) for a complete account on existence and uniqueness in the context of renormalized solutions. Moreover, the case of a bounded zero-order nonlinearity has been treated in [23. Both weak and strong regularity of the, so called, SOLA solutions (solutions obtained as limit of approximations) have been also obtained (see [2] 26, 1 ] and references therein).

Finally, concerning the singular model case, for suitably smooth data $f$ and $\mu$, the existence of solutions to problems as in (1.1) was investigated in [15] (see also [3] 12] 14]).

In this note we consider nonnegative integrable data $f$ and $u_{0}$, a nonnegative bounded Radon measure as nonhmogeneous source and a merely continuous, and possibly singular at the origin, nonlinear zero-order term $h(s)$. Under these general assumptions we prove existence of a nonnegative solution for problem

$$
\begin{cases}u_{t}-\Delta_{p} u=h(u) f+\mu & \text { in } \Omega \times(0, T), \\ u=0 & \text { on } \partial \Omega \times(0, T), \\ u=u_{0} & \text { in } \Omega \times\{0\},\end{cases}
$$

and uniqueness of finite energy solutions (in the homogeneous case) provided $h$ is nonincreasing.

The plan of the paper is as follows: in Section 2 we present our main assumptions and we state the main existence result. Section 3 is devoted to the proof of the existence result; the approximation scheme is presented and the basic a priori estimates are obtained (Section 3.1), then the passage to the limit is performed in Section 3.2 while in Section 3.3 some further regularity issues are discussed. Finally, in Section 4 a uniqueness result is presented.

Notations. The parabolic cylinder is denoted by $Q=\Omega \times(0, T)$ (by $Q_{t}=\Omega \times(0, t)$ for a generic $t>0$ ), while its lateral surface is $\Gamma=\partial \Omega \times(0, T)$. We denote by $\mathcal{M}(Q)$ the space of Radon measures with bounded total variation on $Q$. We will denote with $r^{*}=\frac{r N}{N-r}$ the Sobolev conjugate of $1 \leq r<N$, while $r^{\prime}=\frac{r}{r-1}$ indicates the Hölder conjugate of $r>1$.

For fixed $k>0$ we will made use of the truncation functions $T_{k}$ and $G_{k}$ defined, resp., as $T_{k}(s)=$ $\max (-k, \min (s, k))$, and $G_{k}(s)=(|s|-k)^{+} \operatorname{sign}(s)$. For $\eta, \delta>0$, we define

$$
\tilde{T}_{k, \eta}(s):=\int_{0}^{s} T_{k}^{\eta}(t) d t
$$

and

$$
V_{\delta}(s):= \begin{cases}1 & s \leq \delta \\ \frac{2 \delta-s}{\delta} & \delta<s<2 \delta \\ 0 & s \geq 2 \delta .\end{cases}
$$

For the sake of simplicity we will use the simplified notations

$$
\int_{Q} f=\int_{0}^{T} \int_{\Omega} f=\int_{0}^{T} \int_{\Omega} f(x, t) d x d t
$$

and

$$
\int_{\Omega} f=\int_{\Omega} f(x, t) d x
$$

when referring to integrals when no ambiguity on the variable of integration is possible.

Finally we denote by

$$
\Omega_{\epsilon}:=\{x \in \Omega: \operatorname{dist}(x, \partial \Omega)<\epsilon\},
$$


which is well defined for a sufficiently smooth $\partial \Omega$, say Lipschitz.

If no otherwise specified, we will denote by $C$ several constants whose value may change from line to line and, sometimes, on the same line. These values will only depend on the data but they will never depend on the indexes of the sequences we will often introduce.

\section{Setting AND MAIN EXISTENCE RESULt}

Let $\Omega$ be a bounded and smooth open subset of $\mathbb{R}^{N}(N \geq 2)$, and let $T>0$. We consider the following nonlinear parabolic problem

$$
\begin{cases}u_{t}-\operatorname{div}(a(x, t, \nabla u))=h(u) f+\mu & \text { in } Q, \\ u(x, t)=0 & \text { on } \Gamma, \\ u(x, 0)=u_{0}(x) & \text { in } \Omega,\end{cases}
$$

where $f \geq 0$ belongs to $L^{1}(Q), \mu \geq 0$ belongs to $\mathcal{M}(Q), a(x, t, \xi): \Omega \times(0, T) \times \mathbb{R}^{N} \rightarrow \mathbb{R}^{N}$ is a Carathéodory function satisfying

$$
\begin{aligned}
& a(x, t, \xi) \cdot \xi \geq \alpha|\xi|^{p}, \quad \alpha>0, \\
& |a(x, t, \xi)| \leq \beta|\xi|^{p-1}, \quad \beta>0, \\
& (a(x, t, \xi)-a(x, t, \eta)) \cdot(\xi-\eta)>0,
\end{aligned}
$$

for every $\xi \neq \eta$ in $\mathbb{R}^{N}$ and for almost every $(x, t) \in Q$, with $2-\frac{1}{N+1}<p<N$. A prototype of the operators we consider is the usual $p$-laplacian defined as $-\operatorname{div}\left(|\nabla u|^{p-2} \nabla u\right)$. The initial datum $u_{0}$ is nonnegative and it belongs to $L^{1}(\Omega)$. The bound from below on $p$, even if technical, is standard as it ensures the gradient of the solution to belong to $L^{1}\left(0, T ; L_{\text {loc }}^{1}(\Omega)\right)$. The term $h:[0, \infty) \rightarrow[0, \infty]$ is a continuous and possibly singular function with $h(0) \neq 0$ which it is finite outside the origin and such that

$$
\exists r \geq 0, C, s_{0}>0: h(s) \leq \frac{C}{s^{\gamma}} \text { for all } s \leq s_{0},
$$

and such that $h$ is bounded in $\left[s_{0}, \infty\right)$.

In the following it will be useful the introduction of the notation $\sigma:=\max (1, \gamma)$. Let us give the notion of solution we shall consider from now on

Definition 2.1. A distributional solution of problem $(\mathbb{P})$ is a function $u \in L^{1}\left(0, T ; W_{\text {loc }}^{1,1}(\Omega)\right)$ with both $|a(x, t, \nabla u)|$ and $h(u) f$ belonging to $L^{1}\left(0, T ; L_{\text {loc }}^{1}(\Omega)\right)$, such that

$$
\lim _{\epsilon \rightarrow 0} \frac{1}{\epsilon} \int_{\Omega_{\epsilon}} T_{k}(u)=0 \text { for a.e. } t \in(0, T), \forall k>0,
$$

and

$$
-\int_{Q} u \varphi_{t}-\int_{\Omega} u_{0} \varphi(x, 0)+\int_{Q} a(x, t, \nabla u) \cdot \nabla \varphi=\int_{Q} h(u) f \varphi+\int_{Q} \varphi d \mu,
$$

for every $\varphi \in C_{c}^{1}(\Omega \times[0, T))$.

Remark 2.2. Let us remark that (2.4) is the weak way we recover that $u=0$ on $\Gamma$. Condition (2.4) is known to be weaker of the classical request to have a solution lying in a space with zero Sobolev trace and it allows to unify the discussion of both $\gamma \leq 1$ and $\gamma>1$ (the same was done, in the stationary case, in [31]) and to avoid truncations in the definition. We stress that this is the only point where we exploit the regularity of $\partial \Omega$. If we only assume $\Omega$ to be an open and bounded subset of $\mathbb{R}^{N}$ then everything works fine provided, in the case $\gamma>1$, the boundary condition is intended as $T_{k}^{\frac{\gamma-1+p}{p}}(u) \in L^{p}\left(0, T ; W_{0}^{1, p}(\Omega)\right)$, for any $k>0$ i.e. a suitable power of every truncation of $u$ lies, for almost every $t \in(0, T)$, in a Sobolev space with zero classical trace. If $\gamma \leq 1$ then one may assume $T_{k}(u) \in L^{p}\left(0, T ; W_{0}^{1, p}(\Omega)\right.$ ), for any $k>0$ (this is how, for instance, the boundary condition was given in [6 30] 35]). 
Theorem 2.3. Let a satisfy (2.1), (2.2), (2.3), let h satisfy (․ and suppose that $f \in L^{1}(Q), u_{0} \in L^{1}(\Omega)$, and $\mu \in \mathcal{M}(Q)$ are nonnegative. Then there exists a nonnegative distributional solution u of problem $\mathbb{P}$.

\section{EXISTENCE OF A DISTRIBUTIONAL SOLUTION}

In this section we prove Theorem 2.3 To deduce the existence of a distributional solution we work by approximation. First we introduce the approximating scheme and we get basic a priori estimates on the approximating solutions. Then we pass to the limit, the main difficulty relying in carefully treat the nonlinear term on the set where the approximating solutions vanish and in recovering the boundary datum. At the end of this section we provide some further regularity results on the solution we obtained.

3.1. Approximation scheme and a priori estimates. Consider the following scheme of approximation

$$
\begin{cases}\left(u_{n}\right)_{t}-\operatorname{div}\left(a\left(x, t, \nabla u_{n}\right)\right)=h_{n}\left(u_{n}\right) f_{n}+\mu_{n} & \text { in } Q, \\ u_{n}(x, t)=0 & \text { on } \Gamma, \\ u_{n}(x, 0)=u_{0_{n}}(x) & \text { in } \Omega,\end{cases}
$$

where $h_{n}(s):=T_{n}(h(s)), f_{n}:=T_{n}(f), u_{0_{n}}(x):=T_{n}\left(u_{0}(x)\right)$ and $\mu_{n}$ is a sequence of smooth functions, bounded in $L^{1}(Q)$, that converges in the narrow topology of measures to $\mu$. The existence of such a sequence $\mu_{n}$ is obtained by standard convolution arguments.

First of all we need to show the existence of a weak solution to 3.1). The proof, which is based on the Schauder fixed point theorem, is quite standard but we sketch it for completeness.

Lemma 3.1. Let a satisfy (2.1), (2.2) and 2.3.). Then, for any fixed $n \in \mathbb{N}$, there exists a nonnegative solution $u_{n} \in L^{P}\left(0, T ; W_{0}^{1, p}(\Omega)\right) \cap L^{\infty}(Q)$ such that $\left(u_{n}\right)_{t} \in L^{P^{\prime}}\left(0, T ; W^{-1, p^{\prime}}(\Omega)\right)$ to problem 3.1.

Proof. Let $n \in \mathbb{N}$ be fixed, $v \in L^{P}(Q)$ and consider the following problem

$$
\begin{cases}w_{t}-\operatorname{div}(a(x, t, \nabla w))=h_{n}(v) f_{n}+\mu_{n} & \text { in } Q, \\ w(x, t)=0 & \text { on } \Gamma, \\ w(x, 0)=u_{0_{n}}(x) & \text { in } \Omega .\end{cases}
$$

It follows from classical theory (see [24]) that problem [3.2) admits a unique solution $w \in L^{p}\left(0, T ; W_{0}^{1, p}(\Omega)\right) \cap$ $C\left([0, T] ; L^{2}(\Omega)\right)$ such that $w_{t} \in L^{p^{\prime}}\left(0, T ; W^{-1, p^{\prime}}(\Omega)\right)$ for every fixed $v \in L^{p}(Q)$. Furthermore $w$ belongs to $L^{\infty}(Q)$.

Our aim is to prove the existence of a fixed point for the map

$$
G: L^{P}(Q) \rightarrow L^{P}(Q)
$$

which for any $v \in L^{P}(Q)$ gives the weak solution $w$ to 3.2.

We take $w$ as test function in the weak formulation of 3.2 obtaining

$$
\int_{0}^{T}\left\langle w_{t}, w\right\rangle+\int_{Q} a(x, t, \nabla w) \cdot \nabla w=\int_{Q} h_{n}(v) f_{n} w+\int_{Q} \mu_{n} w .
$$

By 2.1) and by classical integration by parts formula, one has

$$
\frac{1}{2} \int_{\Omega} w^{2}(x, T)-\frac{1}{2} \int_{\Omega} u_{0_{n}}^{2}(x)+\alpha \int_{Q}|\nabla w|^{p} \leq \int_{0}^{T}\left\langle w_{t}, w\right\rangle+\int_{Q} a(x, t, \nabla w) \cdot \nabla w .
$$

For the right hand side of (3.3) by the Hölder inequality

$$
\int_{Q} h_{n}(v) f_{n} w+\int_{Q} \mu_{n} w \leq C n^{2}\left(\int_{Q}|w|^{p}\right)^{\frac{1}{p}}+C\left\|\mu_{n}\right\|_{L^{\infty}(Q)}\left(\int_{Q}|w|^{p}\right)^{\frac{1}{p}}
$$

and so, dropping a positive term

$$
\alpha \int_{Q}|\nabla w|^{p} \leq C\left(n^{2}+\left\|\mu_{n}\right\|_{L^{\infty}(Q)}\right)\left(\int_{Q}|w|^{p}\right)^{\frac{1}{p}}+\frac{1}{2} \int_{\Omega} u_{0_{n}}^{2} .
$$


Poincaré inequality implies for some constant $C$

$$
\int_{Q}|w|^{p} \leq C\left(n^{2}+\left\|\mu_{n}\right\|_{L^{\infty}(Q)}\right)\left(\int_{Q}|w|^{p}\right)^{\frac{1}{p}}+\frac{C}{2} \int_{\Omega} u_{0_{n^{\prime}}}^{2}
$$

which implies

$$
\left(\int_{Q}|w|^{p}\right)^{\frac{1}{p}} \leq C
$$

The constant $C$ is independent of $v$, and so the ball $B:=B_{C}(0)$ of $L^{P}(Q)$ of radius $C$ is invariant for the map $G$.

Now we check the continuity of the map $G$. Let $v_{k}$ be a sequence of functions converging to $v$ in $L^{p}(Q)$.

By the dominated convergence theorem one has that $h_{n}\left(v_{k}\right) f_{n}+\mu_{n}$ converges to $h_{n}(v) f_{n}+\mu_{n}$ in $L^{P}(Q)$. Hence, by uniqueness, one deduces that $w_{k}:=G\left(v_{k}\right)$ converges to $w:=G(v)$ in $L^{p}(Q)$.

Lastly we need $G(B)$ to be relatively compact. Let $v_{k}$ be a bounded sequence, and let $w_{k}=G\left(v_{k}\right)$. Reasoning as to obtain 3.4, we have

$$
\alpha \int_{Q}\left|\nabla w_{k}\right|^{p} \leq C\left(n^{2}+\left\|\mu_{n}\right\|_{L^{\infty}(Q)}\right)\left(\int_{Q}\left|w_{k}\right|^{p}\right)^{\frac{1}{p}}+\frac{1}{2} \int_{\Omega} u_{0_{n}}^{2},
$$

where $C$ is clearly independent from $v_{k}$. Recalling (3.5) this means that $w_{k}$ is bounded with respect to $k$ in $L^{P}\left(0, T ; W_{0}^{1, P}(\Omega)\right)$. We also deduce from the equation that $\left(w_{k}\right)_{t}$ is bounded with respect to $k$ in $L^{p^{\prime}}\left(0, T ; W^{-1, p^{\prime}}(\Omega)\right)$. Hence $w_{k}$ admits a strongly convergent subsequence in $L^{p}(Q)$ (see [36]). This concludes the proof.

In the next lemma we collect the basic estimates on $u_{n}$ which will allow us to pass to the limit in the approximating formulation 3.1.

Lemma 3.2. Let $f \in L^{1}(Q), \mu \in \mathcal{M}(Q), u_{0} \in L^{1}(\Omega)$ be nonnegative, let a satisfy [2.1, 2.2] and (2.3) and let $h$ satisfy (h). Then the sequence $u_{n}$ of solutions of 3.1 is bounded in $L^{\infty}\left(0, T ; L^{1}(\Omega)\right)$ and in $L^{q}\left(0, T ; W_{\text {loc }}^{1, q}(\Omega)\right)$ with $q<p-\frac{N}{N+1}$. Moreover one has

$$
\int_{Q}\left|\nabla T_{k}\left(u_{n}\right)\right|^{p} \varphi^{p} \leq C k, \quad \forall k>0,
$$

for every nonnegative $\varphi \in C_{c}^{1}(\Omega \times[0, T))$.

Proof. For a fixed $t \in(0, T]$ we take $T_{1}^{\sigma}\left(u_{n}\right)$ as a test function in the formulation of $u_{n}$ on $Q_{t}$ (recall that $\sigma:=\max (1, \gamma)) ;$ one has

and then

$$
\begin{aligned}
& \int_{0}^{t}\left\langle\left(u_{n}\right)_{t}, T_{1}^{\sigma}\left(u_{n}\right)\right\rangle+\alpha \sigma \int_{Q_{t}}\left|\nabla T_{1}\left(u_{n}\right)\right|^{p} T_{1}\left(u_{n}\right)^{\sigma-1} \leq \\
& \int_{Q_{t} \cap\left\{u_{n} \leq s_{0}\right\}} f_{n} T_{1}^{\sigma-1}\left(u_{n}\right)+\int_{Q_{t} \cap\left\{u_{n}>s_{0}\right\}} h_{n}\left(u_{n}\right) f_{n} T_{1}^{\sigma}\left(u_{n}\right)+\int_{Q_{t}} \mu_{n} T_{1}^{\sigma}\left(u_{n}\right),
\end{aligned}
$$

$$
\int_{Q_{t}}\left(\tilde{T}_{1, \sigma}\left(u_{n}\right)\right)_{t} \leq\|f\|_{L^{1}(Q)}\left(1+\sup _{s \in\left[s_{0},+\infty\right)} h(s)\right)+\left\|\mu_{n}\right\|_{L^{1}(\Omega)},
$$

where $\tilde{T}_{1, \sigma}(s)$ is defined in $\left[1.2\right.$. Furthermore, observing that $\tilde{T}_{1, \sigma}(s) \geq s-1$, one gets

$$
\int_{\Omega} u_{n}(x, t) \leq\|f\|_{L^{1}(Q)}\left(1+\sup _{s \in\left[s_{0},+\infty\right)} h(s)\right)+\left\|\mu_{n}\right\|_{L^{1}(Q)}+|\Omega|+\int_{\Omega} \tilde{T}_{1, \sigma}\left(u_{0}\right) .
$$

Every term on the right hand side of 3.7 is also bounded with respect to $t$; hence taking the supremum on $t$ we obtain that

$$
\left\|u_{n}\right\|_{L^{\infty}\left(0, T ; L^{1}(\Omega)\right)} \leq C
$$


Now let $\varphi \in C_{c}^{1}(\Omega \times[0, T))$ be nonnegative and take $\left(T_{k}\left(u_{n}\right)-k\right) \varphi^{p}$ as a test function in 3.1

$$
\int_{0}^{T}\left\langle\left(u_{n}\right)_{t},\left(T_{k}\left(u_{n}\right)-k\right) \varphi^{p}\right\rangle+\int_{Q}\left|\nabla T_{k}\left(u_{n}\right)\right|^{p} \varphi^{p}+p \int_{Q} a\left(x, t, \nabla u_{n}\right) \cdot \nabla \varphi \varphi^{p-1}\left(T_{k}\left(u_{n}\right)-k\right) \leq 0,
$$

which gives

$$
\int_{Q}\left|\nabla T_{k}\left(u_{n}\right)\right|^{p} \varphi^{p} \leq \epsilon \int_{Q}\left|\nabla T_{k}\left(u_{n}\right)\right|^{p} \varphi^{p}+C_{\epsilon} k p \int_{Q}|\nabla \varphi|^{p}+C k,
$$

where we also used that

$$
\left|\int_{0}^{T}\left\langle\left(u_{n}\right)_{t},\left(T_{k}\left(u_{n}\right)-k\right) \varphi^{p}\right\rangle\right| \leq\left|\int_{\Omega}\left(\tilde{T}_{k, 1}\left(u_{0_{n}}\right)-k u_{0_{n}}\right) \varphi^{p}(x, 0)\right|+p\left|\int_{Q}\left(\tilde{T}_{k, 1}\left(u_{n}\right)-k u_{n}\right) \varphi^{p-1} \varphi_{t}\right| \leq C k .
$$

Hence one has that

$$
\int_{Q}\left|\nabla T_{k}\left(u_{n}\right)\right|^{p} \varphi^{p} \leq C k
$$

where the constant $C$ does not depend on $n$. By taking $\left(T_{1}\left(G_{k}\left(u_{n}\right)\right)-1\right) \varphi^{p}$ as a test function in 3.1 where $\varphi \in C_{c}^{1}(\Omega \times[0, T))$ one may also deduce

$$
\int_{Q \cap\left\{k<u_{n}<k+1\right\}}\left|\nabla u_{n}\right|^{p} \varphi^{p} \leq C
$$

From (3.8) and 3.9) one can reason as in [4 Theorem 4] in order to deduce that $u_{n}$ is bounded in $L^{q}\left(0, T ; W_{\text {loc }}^{1, q}(\Omega)\right)$ with $q<p-\frac{N}{N+1}$.

The estimates on $u_{n}$ imply that the (possibly) singular term is locally bounded in $L^{1}(Q)$. In fact, we have

Corollary 3.3. Under the assumptions of Lemma 3.2 one has that

$$
\int_{Q} h_{n}\left(u_{n}\right) f_{n} \varphi \leq C
$$

for every nonnegative $\varphi \in C_{C}^{1}(\Omega \times[0, T))$ with $C$ not depending on $n$.

Proof. Let us take $0 \leq \varphi \in C_{c}^{1}(\Omega \times[0, T))$ as a test function in 3.1 obtaining

$$
\int_{Q} h_{n}\left(u_{n}\right) f_{n} \varphi \leq \beta \int_{Q}\left|\nabla u_{n}\right|^{p-1}|\nabla \varphi| \leq C \int_{\operatorname{supp}(\varphi)}\left|\nabla u_{n}\right|^{q}+C
$$

and the right hand side of the previous is bounded thanks to Lemma 3.2

Lemma 3.4. Under the assumptions of Lemma 3.2 there exists $u \in L^{q}\left(0, T ; W_{\text {loc }}^{1, q}(\Omega)\right)$ for any $q<p-\frac{N}{N+1}$ such that, up to a subsequence, $u_{n}$ converges to $u$ a.e. on $Q$, weakly in $L^{q}\left(0, T ; W_{\text {loc }}^{1, q}(\Omega)\right)$ and strongly in $L^{1}\left(0, T ; L_{\text {loc }}^{1}(\Omega)\right)$. Moreover $\nabla u_{n}$ converges almost everywhere to $\nabla u$ in $Q$. In particular $a\left(x, t, \nabla u_{n}\right)$ strongly converges to $a(x, t, \nabla u)$ in $L^{1}\left(0, T ; L_{\text {loc }}^{1}(\Omega)\right)$.

Proof. From Lemma 3.2 we know that $u_{n}$ is bounded in $L^{q}\left(0, T ; W_{\text {loc }}^{1, q}(\Omega)\right)$ with $q<p-\frac{N}{N+1}$. Moreover from Corollary 3.3 one has that the right hand side of 3.1 is bounded in $L^{1}\left(0, T ; L_{\text {loc }}^{1}(\Omega)\right)$. Hence, let $\varphi \in C_{c}^{1}(\Omega)$ then one has that $\left(u_{n} \varphi\right)_{t}$ is bounded in $L^{s}\left(0, T ; W^{-1, s}(\Omega)\right)+L^{1}(Q)$ with $s=\frac{q}{p-1}$, which is sufficient to apply [36 Corollary 4] in order to deduce that $u_{n}$ converges to a function $u$ in $L^{1}\left(0, T ; L_{\text {loc }}^{1}(\Omega)\right)$. Furthermore, since the right hand side of (3.1) is bounded in $L^{1}\left(0, T ; L_{\text {loc }}^{1}(\Omega)\right)$ and $u_{n}$ is bounded in $L^{q}\left(0, T ; W_{\text {loc }}^{1, q}(\Omega)\right)$, one can reason as in the proof of Theorem 4.1 of [5] (see also [2] Theorem 3.3]) deducing that $\nabla u_{n}$ converges to $\nabla u$ almost everywhere in $Q$. The last sentence of the statement can be checked by using (2.2) and Vitali's theorem in order to show that $\left|\nabla u_{n}\right|^{p-1}$ is strongly compact in $L^{1}\left(0, T ; L_{\text {loc }}^{1}(\Omega)\right)$. 
3.2. Passing to the limit. Here, using the results obtained in Section 3.1 we pass to the limit in 3.1 in order to prove Theorem 2.3

Proof of Theorem 2.3 We want to show that $u$, i.e. the almost everywhere limit of $u_{n}$ found in Lemma 3.4 is a solution to $\mathbb{P}$. We first want to obtain (2.5) by passing to the limit in

$$
-\int_{Q} u_{n} \varphi_{t}-\int_{\Omega} u_{0_{n}} \varphi(x, 0)+\int_{Q} a\left(x, t, \nabla u_{n}\right) \cdot \nabla \varphi=\int_{Q} h_{n}\left(u_{n}\right) f_{n} \varphi+\int_{Q} \mu_{n} \varphi,
$$

where $\varphi \in C_{c}^{1}(\Omega \times[0, T))$. Since $u_{n}$ is strongly compact in $L^{1}\left(0, T ; L_{\text {loc }}^{1}(\Omega)\right)$ and using the definition of $u_{0_{n}}$, we easily pass to the limit in the first two terms of the previous equality. We can also pass to the limit in the the third term by using Lemma 3.4 By the definition of $\mu_{n}$ we also pass to the limit in the last term. We are left to pass to the limit in the nonlinear lower order term involving $h$. If $h(0)<\infty$ we use Lebesgue's dominated convergence theorem and we easily pass $n$ to the limit.

Hence, assume $h(0)=\infty$. Let us take $\varphi \geq 0$ (a standard density argument will allow to deduce the result also for sign changing test functions). For $\delta>0$ we split the term as

$$
\int_{Q} h_{n}\left(u_{n}\right) f_{n} \varphi=\int_{Q \cap\left\{u_{n} \leq \delta\right\}} h_{n}\left(u_{n}\right) f_{n} \varphi+\int_{Q \cap\left\{u_{n}>\delta\right\}} h_{n}\left(u_{n}\right) f_{n} \varphi,
$$

Without losing generality we may assume the parameter $\delta$ running outside the set $\{\eta:|\{u(x, t)=\eta\}|>0\}$ which is at most a countable. The second term in (3.11) passes to the limit again by the Lebesgue theorem as

$$
h_{n}\left(u_{n}\right) f_{n} \varphi \chi_{\left\{u_{n}>\delta\right\}} \leq \sup _{s \in[\delta, \infty)}[h(s)] f \varphi \in L^{1}(Q),
$$

namely

$$
\lim _{n \rightarrow \infty} \int_{Q \cap\left\{u_{n}>\delta\right\}} h_{n}\left(u_{n}\right) f_{n} \varphi=\int_{Q \cap\{u>\delta\}} h(u) f \varphi .
$$

Let us observe that using the Fatou lemma and Corollary 3.3 imply $h(u) f \in L^{1}\left(0, T ; L_{\text {loc }}^{1}(\Omega)\right)$. This allows to apply once again the Lebesgue theorem as $\delta \rightarrow 0$ obtaining

$$
\lim _{\delta \rightarrow 0} \lim _{n \rightarrow \infty} \int_{Q \cap\left\{u_{n}>\delta\right\}} h_{n}\left(u_{n}\right) f_{n} \varphi=\int_{Q \cap\{u>0\}} h(u) f \varphi .
$$

We also observe that $h(u) f \in L^{1}\left(0, T ; L_{\text {loc }}^{1}(\Omega)\right)$ gives that the set $\{u=0\}$ is contained in the set $\{f=0\}$ up to a set of zero Lebesgue measure. This means that

$$
\int_{Q \cap\{u>0\}} h(u) f \varphi=\int_{Q} h(u) f \varphi,
$$

and then the proof is done once we have shown that the first term in the right hand side of (3.11) converges to zero as, resp., $n \rightarrow \infty$ and as $\delta \rightarrow 0$. To this aim we take $V_{\delta}\left(u_{n}\right) \varphi\left(V_{\delta}\right.$ is defined in (1.3) ) as test function in 3.1. Dropping a negative term, one has

$$
\begin{aligned}
\int_{Q \cap\left\{u_{n} \leq \delta\right\}} h_{n}\left(u_{n}\right) f_{n} \varphi & \leq \int_{0}^{T}\left\langle\left(u_{n}\right)_{t}, V_{\delta}\left(u_{n}\right) \varphi\right\rangle+\int_{Q}\left|\nabla u_{n}\right|^{p-1}|\nabla \varphi| V_{\delta}\left(u_{n}\right) \\
& \leq-\int_{\Omega} \Phi_{\delta}\left(u_{n}\right) \varphi_{t}+\int_{Q}\left|\nabla u_{n}\right|^{p-1}|\nabla \varphi| V_{\delta}\left(u_{n}\right),
\end{aligned}
$$

where $\Phi_{\delta}(s)=\int_{0}^{s} V_{\delta}(t) d t$. Furthermore, from the fact that $\int_{\Omega} \Phi_{\delta}\left(u_{n}\right) \leq \delta$ and from (3.6), one is able to deduce that

which implies that

$$
\limsup _{n \rightarrow \infty} \int_{Q \cap\left\{u_{n} \leq \delta\right\}} h_{n}\left(u_{n}\right) f_{n} \varphi \leq C \delta^{\frac{1}{p^{\prime}}},
$$

$$
\lim _{\delta \rightarrow 0} \limsup _{n \rightarrow \infty} \int_{Q \cap\left\{u_{n} \leq \delta\right\}} h_{n}\left(u_{n}\right) f_{n} \varphi=0 .
$$


Hence, we deduce that

$$
\lim _{n \rightarrow \infty} \int_{Q} h_{n}\left(u_{n}\right) f_{n} \varphi=\int_{Q} h(u) f \varphi
$$

for every $\varphi \in C_{c}^{1}(\Omega \times[0, T))$ and this proves that $u$ satisfies $[2.5$.

In order to conclude the proof of Theorem 2.3 we show that the boundary condition holds; namely $u$ satisfies 2.4. By taking $T_{k}^{\sigma}\left(u_{n}\right)(k>0)$ as a test function in 3.1) one can deduce that $T_{k}^{\frac{\sigma-1+p}{p}}\left(u_{n}\right)$ is bounded in $L^{P}\left(0, T ; W_{0}^{1, P}(\Omega)\right)$ with respect to $n$. Hence one has that, for almost every $t \in(0, T)$ and for every $k>0$, $T_{k}^{\frac{\sigma-1+p}{p}}(u(x, t)) \in W_{0}^{1, p}(\Omega)$. If $\gamma \leq 1$ then $T_{k}(u(x, t)) \in W_{0}^{1, p}(\Omega)$ is known to imply (2.4). Otherwise, if $\gamma>1$, one reasons as follows

$$
\frac{1}{\epsilon} \int_{\Omega_{\epsilon}} T_{k}(u) \leq \frac{1}{\epsilon}\left(\int_{\Omega_{\epsilon}} T_{k}^{\frac{\gamma-1+p}{p}}(u)\right)^{\frac{p}{\gamma-1+p}}\left|\Omega_{\epsilon}\right|^{\frac{\gamma-1}{\gamma-1+p}}=\left(\frac{\left|\Omega_{\epsilon}\right|}{\epsilon}\right)^{\frac{\gamma-1}{\gamma-1+p}}\left(\frac{1}{\epsilon} \int_{\Omega_{\epsilon}} T_{k}^{\frac{\gamma-1+p}{p}}(u)\right)^{\frac{p}{\gamma-1+p}},
$$

and taking $\epsilon \rightarrow 0$ in the previous one has that 2.4 holds also in this case. This concludes the proof.

3.3. Some comments on the regularity of the solutions. In this section we show that the scheme of approximation 3.1 can take to a solution $u \in L^{p}\left(0, T ; W_{0}^{1, p}(\Omega)\right)$ under suitable assumptions on the data, and in particular on the nonlinearity $h$ giving rise to some regularizing effects with respect to the purely quasilinear case (i.e. $h \equiv 1$ ). For a general nonhomogeneous datum $\mu$ then no solution belonging to the natural energy space are expected, since only truncations of them are (see 30 31] for similar considerations in the stationary case). So that we restrict to the case $\mu \equiv 0$.

We assume the following control on $h$ at infinity

$$
\exists \theta \geq 0, C, s_{1}>0: h(s) \leq \frac{C}{s^{\theta}} \text { for all } s \geq s_{1}
$$

On the datum $f$ we assume that

$$
\begin{array}{ll}
f \in L^{\frac{p}{p-1+\theta}}\left(0, T ; L\left(\frac{p^{*}}{1-\theta}\right)^{\prime}(\Omega)\right) & \text { if } \theta<1, \\
f \in L^{1}(Q) & \text { if } \theta \geq 1 .
\end{array}
$$

Lemma 3.5. Let a satisfy 2.1, 2.2, 2.3, let $h$ satisfy (h) with $\gamma \leq 1$ and 3.12, let $f$ nonnegative satisfy 3.13; finally let $u_{0} \in L^{2}(Q)$ be nonnegative. Then the solution to $\mathbb{P}$ (with $\mu \equiv 0$ ) found in Theorem 2.3 belongs to $L^{P}\left(0, T ; W_{0}^{1, p}(\Omega)\right)$.

Proof. We need to prove that under our assumptions we can show better a priori estimates on the sequence $u_{n}$, i.e. a solution to 3.1. Hence we take $u_{n}$ as a test function in 3.1 obtaining

$$
\begin{aligned}
\alpha \int_{Q}\left|\nabla u_{n}\right|^{p} & \leq \max _{s \in\left[0, s_{1}\right]}[h(s) s] \int_{Q \cap\left\{u_{n} \leq s_{1}\right\}} f_{n}+\int_{Q \cap\left\{u_{n}>s_{1}\right\}} f_{n} u_{n}^{1-\theta}+\frac{1}{2} \int_{\Omega} u_{0}^{2} \\
& \leq C+\int_{Q \cap\left\{u_{n}>s_{1}\right\}} f_{n} u_{n}^{1-\theta}
\end{aligned}
$$

and hence if $\theta \geq 1$ the estimate is done since

$$
\int_{Q \cap\left\{u_{n}>s_{1}\right\}} f_{n} u_{n}^{1-\theta} \leq \int_{Q \cap\left\{u_{n}>s_{1}\right\}} f_{n} s_{1}^{1-\theta} \leq C .
$$

Otherwise we apply the Hölder, the Sobolev (with constant $\mathcal{S}_{p}$ ) and the Young inequalities to have

$$
\begin{aligned}
\alpha \int_{Q}\left|\nabla u_{n}\right|^{p} & \leq \int_{0}^{T}\|f\|_{L\left(\frac{p^{*}}{1-\theta}\right)_{(\Omega)}^{\prime}}\left\|u_{n}\right\|_{L^{p^{*}}(\Omega)}^{1-\theta} \leq \mathcal{S}_{p}^{1-\theta} \int_{0}^{T}\|f\|_{L\left(\frac{p^{*}}{1-\theta}\right)_{(\Omega)}^{\prime}}\left(\int_{\Omega}\left|\nabla u_{n}\right|^{p}\right)^{\frac{1-\theta}{p}} \\
& \leq C_{\epsilon} \mathcal{S}_{p}^{1-\theta} \int_{0}^{T} \|\left. f\right|_{L\left(\frac{p^{*}}{1-\theta}\right)_{(\Omega)}^{\prime}} ^{\frac{p}{p-1+\theta}}+\epsilon \mathcal{S}_{p}^{1-\theta} \int_{Q}\left|\nabla u_{n}\right|^{p}
\end{aligned}
$$


and taking $\epsilon$ sufficiently small the estimate is closed also if $\theta<1$. Hence $u_{n}$ is bounded in $L^{P}\left(0, T ; W_{0}^{1, p}(\Omega)\right)$ and so $u$ belongs to $L^{P}\left(0, T ; W_{0}^{1, P}(\Omega)\right)$.

Remark 3.6. We underline that requiring that $h$ goes to zero as in (3.12) provides a strong regularizing effect on the Sobolev regularity of the solution to $(\mathrm{P})$. Indeed when $h \equiv 1$ and $\mu \equiv 0$ then $u$ is, in general, expected to have finite energy for a datum $f \in L^{p^{\prime}}\left(0, T ; L^{\left(p^{*}\right)^{\prime}}(\Omega)\right)$ (i.e. $\theta=0$ in (3.12) (see [24]), while for more general (e.g. merely integrable) data then $u$ needs not to (see [4). In our case, even if $h$ is allowed to mildly blow up at the origin $(\gamma \leq 1)$, we are able to find more regular solution thanks to the regularizing effect given by the vanishing rate of $h$ at infinity. This improved regularity is a key tool for uniqueness, since, as we will see in the next section, a unique solution can be proven to exist in a suitable subclass of this energy space. Finally we underline that the exponent $\left(\frac{p^{*}}{1-\theta}\right)^{\prime}$ is the same obtained in [6] when $p=2$ while studying the regularizing effect of the singular nonlinearity in the stationary case.

\section{UNIQUENESS OF FINITE ENERGY SOLUTIONS}

In this section we prove uniqueness of finite energy solutions to

$$
\begin{cases}u_{t}-\operatorname{div}(a(x, t, \nabla u))=h(u) f & \text { in } Q, \\ u(x, t)=0 & \text { on } \Gamma, \\ u(x, 0)=u_{0}(x) & \text { in } \Omega ;\end{cases}
$$

provided $h$ is nonincreasing. We say that a distributional solution $u$ of problem $\left[\mathrm{P}_{0}\right.$ is said to have finite energy provided $u \in L^{p}\left(0, T ; W_{0}^{1, p}(\Omega)\right)$ and $u_{t} \in L^{p^{\prime}}\left(0, T ; W^{-1, p^{\prime}}(\Omega)\right)+L^{1}(Q)$. We have the following

Theorem 4.1. Let $h$ be nonincreasing, then there is at most one finite energy solution to $\left(\mathrm{P}_{0}\right]$.

The first step consists in extending the set of admissible test functions in (2.5), namely we have

Lemma 4.2. Let $u$ be a finite energy solution to $\left[\mathrm{P}_{0}\right.$ then u satisfies

$$
\int_{0}^{T}\left\langle\left(u_{t}\right)_{1}, \varphi\right\rangle+\int_{Q}\left(u_{t}\right)_{2} \varphi+\int_{Q} a(x, t, \nabla u) \cdot \nabla \varphi=\int_{Q} h(u) f \varphi,
$$

where $\left(u_{t}\right)=\left(u_{t}\right)_{1}+\left(u_{t}\right)_{2}$ with $\left(u_{t}\right)_{1} \in L^{p^{\prime}}\left(0, T ; W^{-1, p^{\prime}}(\Omega)\right),\left(u_{t}\right)_{2} \in L^{1}(Q)$ and for every $\varphi \in L^{P}\left(0, T ; W_{0}^{1, p}(\Omega)\right) \cap$ $L^{\infty}(Q)$.

Proof. Let $\varphi \in L^{P}\left(0, T ; W_{0}^{1, P}(\Omega)\right) \cap L^{\infty}(Q)$ be a nonnegative function and consider a sequence $\varphi_{n}$ of smooth nonnegative functions converging to $\varphi$ in $L^{p}\left(0, T ; W_{0}^{1, p}(\Omega)\right)$, without loss of generality one may assume $\left\|\varphi_{n}\right\|_{L^{\infty}(Q)} \leq\|\varphi\|_{L^{\infty}(Q)}$. Now we take $\varphi_{n}$ in (2.5) (recall that $\mu \equiv 0$ ) and we integrate by parts (see for instance [9]) in order to obtain

$$
\int_{0}^{T}\left\langle\left(u_{t}\right)_{1}, \varphi_{n}\right\rangle+\int_{Q}\left(u_{t}\right)_{2} \varphi_{n}+\int_{Q} a(x, t, \nabla u) \cdot \nabla \varphi_{n}=\int_{Q} h(u) f \varphi_{n} .
$$

Due to the definition of $\varphi_{n}$ and on the regularity of $u$ one can pass to the limit in the left hand side of the previous equality; in particular $h(u) f \varphi_{n}$ is bounded in $L^{1}(Q)$ and by Fatou's lemma one obtains that also $h(u) f \varphi \in L^{1}(Q)$. Using dominated convergence theorem one can pass to the limit also on the right hand side of 4.2 and we conclude.

Proof of Theorem 4.1 Let $v, w$ be two finite energy solutions of $\left[\mathbb{P}_{0}\right]$, and take $T_{k}(v-w) \phi(t)$ in the difference of formulations (4.1) solved by $v, w$, where we define $\phi(t)=\frac{-t}{T}+1$ for $t \in(0, T]$. Using [2.3) and the assumption on $h$ we obtain that

$$
\int_{0}^{T}\left\langle\left(v_{t}\right)_{1}-\left(w_{t}\right)_{1}, T_{k}(v-w) \phi(t)\right\rangle+\int_{Q}\left(\left(v_{t}\right)_{2}-\left(w_{t}\right)_{2}\right) T_{k}(v-w) \phi(t) \leq 0 .
$$


It follows from of [17 Lemma 7.1] that $\left(\tilde{T}_{k, 1}(s)\right.$ is defined in [1.2)

$$
\frac{1}{T} \int_{Q} \tilde{T}_{k, 1}(v-w)=0
$$

that, due to the arbitrariness of $k$ and recalling that $v-w \in C\left([0, T] ; L^{1}(\Omega)\right)$, implies that $v(\tau)=w(\tau)$ for any $\tau \in(0, T]$ and for almost every $x$ in $\Omega$ and this concludes the proof.

Remark 4.3. We want to highlight some cases in which the solution to $\mathrm{P}_{0}$ has finite energy. First of all observe that if $h(0)<\infty$ then Lemma 3.5 gives some instances of a finite energy solutions (since, from the equation, one has $\left.u_{t} \in L^{p^{\prime}}\left(0, T ; W^{-1, p^{\prime}}(\Omega)\right)+L^{1}(Q)\right)$. Moreover if we restrict to data $a$ and $f$ not depending on $t$, and we consider $u_{0}=w(x)$ where $w(x)$ is a solution to the associated elliptic problem

$$
\begin{cases}-\operatorname{div}(a(x, \nabla w))=h(w) f & \text { in } \Omega, \\ w=0 & \text { on } \partial \Omega,\end{cases}
$$

then, if $w \in W_{0}^{1, p}(\Omega)$ (see [22 6 31]), one has, by Lemma 4.2 that $u(x, t)=w(x)$ turns out to be a finite energy solution of $\left[\mathrm{P}_{0}\right.$.

Also in the general case of a nonlinearity $h$ blowing up at zero non-trivial non-stationary finite energy solutions do exist as the following example shows

Example 1. We present a case where $h$ actually blows up at the origin and the solution to $\mathrm{P}_{0}$ has finite energy. Let $\gamma \leq 1$ and, for the sake of simplicity let $p=2$, we consider the following problem

$$
\begin{cases}u_{t}-\Delta u=f & \text { in } Q, \\ u(x, t)=0 & \text { on } \Gamma, \\ u(x, 0)=u_{0}(x) & \text { in } \Omega,\end{cases}
$$

where $0 \leq f \in L^{2}\left(0, T ; L^{\left(2^{*}\right)^{\prime}}(\Omega)\right)$ and $0 \leq u_{0} \in L^{2}(\Omega)$. By the classical theory of parabolic equations one has that a positive solution $u \in L^{2}\left(0, T ; W_{0}^{1,2}(\Omega)\right)$ does exist. We observe that $u$ also solves

$$
\begin{cases}u_{t}-\Delta u=\frac{g}{u^{\nu}} & \text { in } Q, \\ u(x, t)=0 & \text { on } \Gamma, \\ u(x, 0)=u_{0}(x) & \text { in } \Omega,\end{cases}
$$

where $g=f u^{\gamma} \in L^{1}(Q)$. Indeed, applying the Hölder and the Young inequalities, one has

$$
\int_{Q} f u^{\gamma} \leq \int_{0}^{T}\|f\|_{L\left(\frac{2^{*}}{\gamma}\right)^{\prime}(\Omega)}\|u\|_{L^{2^{*}}(\Omega)}^{\nu} \leq \int_{0}^{T}\|f\|_{\left(\frac{2{ }^{*}}{\gamma}\right)^{\prime}(\Omega)}^{\frac{2}{2-\gamma}}+\int_{0}^{T}\|u\|_{L^{2^{*}}(\Omega)}^{2}
$$

A similar calculation gives the existence of a finite energy solution even if $\gamma>1$ provided we restrict to a datum $f \in L^{2}\left(0, T ; L^{\left(\frac{2 *}{\gamma}\right)^{\prime}}(\Omega)\right)$. Finally we also underline that in case $h(s) \approx s^{-\gamma}$ near $s=0$ with $h(s) \geq c$ for all $s \geq 0$ then a finite energy solution to $\mathrm{P}_{0}$ also exists, using that $g=\frac{f}{h(u)} \leq \frac{f}{c}$.

Remark 4.4. For simplicity we presented our uniqueness result in the homogeneous case $\mu \equiv 0$; as we have seen the key role in the proof is played by the possibility to extend the set of test functions (Lemma 4.2). For this reason, with the same proof, one can allow also nonhomogeneous measure data $\mu \in L^{1}(Q)+L^{P^{\prime}}\left(0, T ; W^{-1, p^{\prime}}(\Omega)\right)$; as showed in [33 Proposition 3.1] (see also [25] Theorem 2.6]) measure with such a structure are absolutely continuous with respect to the parabolic $p$-capacity.

\section{ACKNOWLEDGEMENTS}

The authors are partially supported by the Gruppo Nazionale per l'Analisi Matematica, la Probabilità e le loro Applicazioni (GNAMPA) of the Istituto Nazionale di Alta Matematica (INdAM). 


\section{REFERENCES}

[1] P. Baroni, A. Di Castro, G. Palatucci, Global estimates for nonlinear parabolic equations, J. Evol. Equ., 13 (1) (2013), 163-195.

[2] L. Boccardo, A. Dall'Aglio T. Gallouët, L. Orsina, Nonlinear Parabolic Equations with Measure Data, J. Funct. Anal., 147 (1997), 237-258.

[3] L. Boccardo, M. Escobedo, M. M. Porzio, Parabolic equations with singular and supercritical reaction terms, Differential Integral Equations, 28 (11-12) (2015), 1155-1172.

[4] L. Boccardo, T. Gallouët, Nonlinear elliptic and parabolic equations involving measure data, J. Funct. Anal., 87 (1989), 149-169.

[5] L. Boccardo, F. Murat, Almost everywhere convergence of the gradients of solutions to elliptic and parabolic equations, Nonlinear Anal., 19 (6) (1992), 581-597.

[6] L. Boccardo, L. Orsina, Semilinear elliptic equations with singular nonlinearities, Calc. Var. and PDEs, 37 (2010), 363-380.

[7] B. Bougherara, J. Giacomoni, J. Hernández, Existence and regularity of weak solutions for singular elliptic problems, Proceedings of the 2014 Madrid Conference on Applied Mathematics in honor of Alfonso Casal, 19-30, Electron. J. Differ. Equ. Conf., 22, Texas State Univ., San Marcos, TX, 2015.

[8] A. Canino, B. Sciunzi, A. Trombetta, Existence and uniqueness for $p$-Laplace equations involving singular nonlinearities, NoDEA Nonlinear Differential Equations Appl., (2016) 23:8.

[9] J. Carrillo, P. Wittbold, Uniqueness of Renormalized Solutions of Degenerate Elliptic-Parabolic Problems, Journal of Differential Equations, 156 (1) (1999), 93-121,

[10] M. G. Crandall, P. H. Rabinowitz, L. Tartar, On a dirichlet problem with a singular nonlinearity, Comm. Part. Diff. Eq., 2 (1977), 193-222.

[11] A. Dall'Aglio, L. Orsina, Nonlinear parabolic equations with natural growth conditions and L1 data, Nonlinear Anal., 27 (1996), 59-73.

[12] I. De Bonis, L. M. De Cave, Degenerate parabolic equations with singular lower order terms, Differential and Integral Equations, 27 (2014), 949-976

[13] L. M. De Cave, F. Oliva, Elliptic equations with general singular lower order term and measure data, Nonlinear Anal., 128 (2015), 391-411.

[14] I. De Bonis, D. Giachetti, Singular parabolic problems with possibly changing sign data, Discrete and Continuous Dynamical Systems - Series B, 19 (7) (2014), 2047-2064.

[15] I. De Bonis, D. Giachetti, Nonnegative solutions for a class of singular parabolic problems involving p-Laplacian, Asymptotic Analysis, 91 (2) (2015), 147-183.

[16] J. Droniou, A. Porretta, A. Prignet, Parabolic capacity and soft measures for nonlinear equations, Potential Anal., 19 (2) (2003), 99-161.

[17] J. Droniou, A. Prignet, Equivalence between entropy and renormalized solutions for parabolic equations with smooth measure data, NoDEA Nonlinear Differential Equations Appl., 14 (1-2) (2007), 181-205

[18] W. Fulks, J. S. Maybee, A Singular Non-Linear Equation, Osaka Journal Mathematics, 12 (1960), 1-19.

[19] J. A. Gatica, V. Oliker, P. Waltman, Singular nonlinear boundary-value problems for second-order ordinary differential equations, Journal of Differential Equations, 79 (1989), 62-78.

[20] D. Giachetti, P.J. Martínez-Aparicio, F. Murat, A semilinear elliptic equation with a mild singularity at $u=0$ : Existence and homogenization, J. Math. Pures Appl., 107 (2017), 41-77.

[21] D. Giachetti, P.J. Martínez-Aparicio, F. Murat, Definition, existence, stability and uniqueness of the solution to a semilinear elliptic problem with a strong singularity at $u=0$, Ann. Sc. Norm. Sup. Pisa (5), 18 (4) (2018), 1395-1442.

[22] A. C. Lazer, P. J. McKenna, On a singular nonlinear elliptic boundary-value problem, Proc. Amer. Math. Soc., 111 (1991), $721-730$.

[23] Z. Liqin, Z. Junning, Existence and uniqueness of renormalized solutions for a class of degenerate parabolic equations, Acta Mathematica Scientia, 29 (2) (2009), 251-264.

[24] J.-L. Lions, Quelques méthodes de résolution des problémes aux limites non linéaire, Dunod et Gautier-Villars, (1969).

[25] T. Klimsiak, A. Rozkosz, On the structure of diffuse measures for parabolic capacities, arXiv:1808.06422

[26] T. Kuusi, G. Mingione, The Wolff gradient bound for degenerate parabolic equations. J. Eur. Math. Soc. (JEMS), 16 (4) (2014), 835-892.

[27] A. Nachman, A. Callegari, A nonlinear singular boundary value problem in the theory of pseudoplastic fluids, SIAM J. Appl. Math., 28 (1986), 271-281.

[28] P. Nowosad, On the integral equation $k f=1 / f$ arising in a problem in communication, J. Math. Appl., 14 (1966), 484-492.

[29] L. Orsina, F. Petitta, A Lazer-McKenna type problem with measures, Differential and Integral Equations, 29 (1-2) (2016), 19-36

[30] F. Oliva, F. Petitta, On singular elliptic equations with measure sources, ESAIM Control Optim. Calc. Var., 22 (1) (2016), 289-308.

[31] F. Oliva, F. Petitta, Finite and Infinite energy solutions of singular elliptic problems: Existence and Uniqueness, Journal of Differential Equations, 264 (2018), 311-340.

[32] F. Petitta, Renormalized solutions of nonlinear parabolic equations with general measure data, Ann. Mat. Pura Appl. (4), 187 (4) (2008), 563-604

[33] F. Petitta, A. Ponce, A. Porretta, Diffuse measures and nonlinear parabolic equations, J. Evol. Equations, 11 (4) (2011), 861-905. 
[34] F. Petitta, A. Porretta, On the notion of renormalized solution to nonlinear parabolic equations with general measure data, J. of Elliptic and Parabolic Equations, 1 (2015), 201-214.

[35] A. Porretta, Existence results for nonlinear parabolic equations via strong convergence of truncations, Ann. Mat. Pura Appl., 177 (1) (1999), 143-172.

[36] J. Simon, Compact sets in the space $L^{p}(0, T ; B)$, Ann. Mat. Pura Appl., 146 (1987), 65-96.

[37] C. A. Stuart, Existence and approximation of solutions of non-linear elliptic equations, Math. Z., 147 (1976), $53-63$.

[38] Y. Sun, D. Zhang, The role of the power 3 for elliptic equations with negative exponents, Calc. Var. Partial Differential Equations, 49 (3-4) (2014), 909-922.

(F. Oliva) Istituto Nazionale di Alta Matematica (Indam), Dipartimento di Scienze di Base e Applicate per l' Ingegneria, "Sapienza" Università DI Roma, Via Scarpa 16, o0161 Roma, Italy, fRanCESCO.0liva@Sbal.UNIROMa1.IT

(F. Petitta) Dipartimento di Scienze di Base e Applicate per l' Ingegneria, "Sapienza" Università di Roma, Via Scarpa 16, o0161 Roma, Italy, fRANCESCO.PETItTA@SbaI.UNIROMA1.IT 